\title{
Dynamical Dipole mode in heavy-ion fusion reactions by using stable and radioactive beams
}

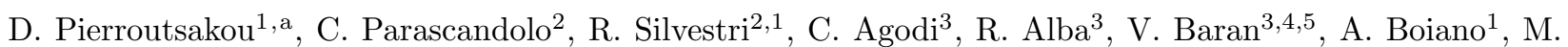
Colonna $^{3}$, R. Coniglione ${ }^{3}$, E. De Filippo ${ }^{6}$, A. De Rosa ${ }^{2,1}$, A. Del Zoppo ${ }^{3}$, M. Di Toro ${ }^{3,7}$, U. Emanuele ${ }^{8}$, F. Farinon $^{9}$, A. Guglielmetti ${ }^{10}$, G. Inglima ${ }^{2,1}$, M. La Commara ${ }^{2,1}$, C. Maiolino ${ }^{3}$, B. Martin ${ }^{2,1}$, M. Mazzocco ${ }^{11}$, C. Mazzocchi $^{10}$, P. Molini ${ }^{11}$, C. Rizzo ${ }^{3,7}$, M. Romoli ${ }^{1}$, M. Sandoli ${ }^{2,1}$, D. Santonocito ${ }^{3}$, C. Signorini ${ }^{11}$, F. Soramel ${ }^{11}$, A. Trifirò ${ }^{8}$, and M. Trimarchi ${ }^{8}$

1 INFN, Sezione di Napoli, via Cinthia, I-80126 Napoli, Italy

2 Dipartimento di Scienze Fisiche, Università di Napoli "Federico II", via Cinthia, I-80126 Napoli, Italy

3 INFN, Laboratori Nazionali del Sud, via Santa Sofia, I-95123 Catania, Italy

4 University of Bucharest, Bucharest,Romania

5 NIPNE-HH, 077125 Magurele, Romania

6 INFN, Sezione di Catania, I-95123 Catania, Italy

7 Dipartimento di Fisica, Università di Catania, I-95123 Catania, Italy

8 INFN, Gruppo Collegato di Messina and Università di Messina, Messina, Italy

9 GSI, Planckstrasse 1, D-64291, Darmstadt, Germany

10 INFN, Sezione di Milano and Dipartimento di Fisica, Univ. di Milano, via Celoria 16, I-20133 Milano, Italy

11 INFN, Sezione di Padova and Dip. di Fisica, Univ. di Padova, via F.Marzolo 8, I-35131, Padova, Italy

\begin{abstract}
The existence of the dynamical dipole mode in the ${ }^{192} \mathrm{~Pb}$ composite system was investigated through the study of its prompt $\gamma$ decay employing the ${ }^{40} \mathrm{Ca}+{ }^{152} \mathrm{Sm}$ and ${ }^{48} \mathrm{Ca}+{ }^{144} \mathrm{Sm}$ reactions at $\mathrm{E}_{l a b}=11$ and $10.1 \mathrm{MeV} / \mathrm{u}$, respectively. The $\gamma$-rays and light charged particles were detected in coincidence with evaporation residues and fission fragments. First results of this experiment show that the dynamical dipole mode survives in collisions involving heavier mass reaction partners than those studied previously. As a fast cooling mechanism on the fusion path, the prompt dipole $\gamma$ radiation could be of interest for the synthesis of super-heavy elements through "hot" fusion reactions. Furthermore, by using radioactive beams and the prompt $\gamma$ radiation as a probe we could get information on the symmetry energy at sub-saturation densities.
\end{abstract}

\section{Introduction}

The dynamical dipole mode is a large amplitude collective oscillation of protons against neutrons that develops along the symmetry axis of the dinuclear system formed in charge asymmetric heavy-ion collisions [1]. It decays emitting prompt $\gamma$ radiation with the following characteristics: (i) it appears as an extra yield in the $\gamma$-ray energy spectra at a centroid energy lower than that of the Giant Dipole Resonance (GDR) thermally excited in the compound nucleus $(\mathrm{CN})$ because it originates from a highly deformed dinuclear system; (ii) an anisotropic angular distribution due to the fact that the oscillation is confined in the reaction plane; (iii) an intensity depending on the reaction dynamics and thus on the incident energy [2].

Since the prompt $\gamma$-ray emission related to the dynamical dipole mode may become comparable to the GDR statistical $\gamma$ emission, it could represent an interesting cooling mechanism of the composite system

\footnotetext{
a e-mail: pierroutsakou@na.infn.it
}

to facilitate the formation of super-heavy elements through "hot" fusion reactions. Super-heavy elements can be formed at low excitation energy because (1) smaller excitation energies have smaller fission probabilities and (2) the shell corrections, responsible for the stability of super-heavy nuclei, decrease with excitation energy. It was estimated in $[3,4]$, in the framework of an "hybrid" statistical model of the CN decay, that the lowering of the $\mathrm{CN}$ excitation energy by an amount ranging between 10 and $15 \mathrm{MeV}$, the typical energy removed by a pre-equilibrium dipole photon, results in an increase of its survival probability against fission and thus in an increase of the evaporation residue cross section. However, in order to predict evaporation residue cross sections of super-heavy elements in charge asymmetric reactions, a realistic theoretical model that follows the dynamical evolution of the system in the multi-dimensional potential energy landscape through quasi-fission or formation of the $\mathrm{CN}$ and its subsequent evaporation and fission including the pre-equilibrium dipole $\gamma$-ray emission in 
the early stages of the collision is needed. Unfortunately, to our knowledge such a model does not exist at the moment. Furthermore, the usefulness of the dynamical dipole mode in the super-heavy element formation could be compromised if the dynamical dipole $\gamma$ yield decreases in collisions involving heavy mass partners as predicted in [5]. Thus, the existence of the dynamical dipole mode in those systems should be investigated as a first step.

From an experimental point of view, the dynamical dipole mode has been observed in charge asymmetric heavy-ion deep inelastic [6-8] and fusion reactions $[7,9,10]$ while the first systematic study of its features (centroid energy, width, intensity) as a function of the incident energy was performed in our previous campaign of experiments [11-14] where compound nuclei in the ${ }^{132} \mathrm{Ce}$ mass region were created. In those measurements, the ${ }^{132} \mathrm{Ce} \mathrm{CN}$ was formed through different charge asymmetry entrance channels at identical excitation energy and with identical spin distribution by using two reaction pairs: ${ }^{32,36} \mathrm{~S}+{ }^{100,96} \mathrm{Mo}$ at $\mathrm{E}_{l a b}=6$ and $9 \mathrm{MeV} / \mathrm{u}$ and ${ }^{36,40} \mathrm{Ar}+{ }^{96,92} \mathrm{Zr}$ at $\mathrm{E}_{l a b}=$ $16 \mathrm{MeV} / \mathrm{u}$. The dynamical dipole mode was evidenced through the observation of an excess of yield in the $\gamma$ ray energy spectrum of the more charge asymmetric system at a centroid energy lower than that of the GDR and with an highly anisotropic angular distribution around $90^{\circ}$ with respect to the beam direction $[13,14]$. The extracted dynamical dipole $\gamma$ yield integrated over energy and over solid angle showed a maximum at $\mathrm{E}_{l a b}=9 \mathrm{MeV} / \mathrm{u}$ [14]. Recently, new results [15] appeared on the dynamical dipole mode excited in the same compound nucleus employing the ${ }^{16} \mathrm{O}+{ }^{116} \mathrm{Sn}$ charge asymmetric reaction at $E_{l a b}=8$ and $15.6 \mathrm{MeV} / \mathrm{u}$. The comparison of the two data sets $[14$, $15]$ with each other and with the theoretical predictions proves that further investigation is needed, from both the theoretical and the experimental point of view, to shed light on the interplay between the different parameters that influence the dynamical dipole mode features.

\section{Search for the dynamical dipole mode in the ${ }^{192} \mathbf{P b}$ mass region}

As explained previously, the prompt dipole $\gamma$ yield is predicted [5] to decrease in collisions involving heavy mass partners. That occurs because reactions with small nuclei are less damped than those involving more nucleons. In order to verify experimentally if this preequilibrium effect survives in heavier systems than those studied up to now, we extended our investigation of the dynamical dipole mode in the mass region of the ${ }^{192} \mathrm{~Pb} \mathrm{CN}$ employing the ${ }^{40} \mathrm{Ca}+{ }^{152} \mathrm{Sm}$ and ${ }^{48} \mathrm{Ca}+{ }^{144} \mathrm{Sm}$ reactions at $\mathrm{E}_{l a b}=440 \mathrm{MeV}$ and $485 \mathrm{MeV}$, respectively. The reactions were performed by using the pulsed beam of ${ }^{40} \mathrm{Ca}$ and ${ }^{48} \mathrm{Ca}$ delivered by the Superconducting Cyclotron of the Laboratori Nazionali del Sud (Italy). The beam impinged on a $1 \mathrm{mg} / \mathrm{cm}^{2}$ thick ${ }^{152} \mathrm{Sm}$ and ${ }^{144} \mathrm{Sm}$ target. The above reactions form the same $\mathrm{CN},{ }^{192} \mathrm{~Pb}$, with identical spin distribution $\left(\mathrm{L}_{\max }=74 \hbar\right)$ according to PACE2 calculations [16] at an excitation energy of $236 \mathrm{MeV}$, evaluated using the empirical formula of ref. [17]. As a first approach, we analyzed the proton energy spectra collected at $\theta_{l a b}=160^{\circ}$ with respect to the beam direction, where emission from solely the $\mathrm{CN}$ is expected and the claim of identical average excitation energy of the $\mathrm{CN}$ in the two reactions was confirmed. However, this quantity will be extracted experimentally by analyzing the energy spectra of the light charged particles detected at different polar angles as in our previous works $[13,14]$. While the entrance channel mass asymmetries of the considered reactions are similar with each other, namely 0.22 and 0.18 for the ${ }^{40} \mathrm{Ca}+{ }^{152} \mathrm{Sm}$ and ${ }^{48} \mathrm{Ca}+{ }^{144} \mathrm{Sm}$ reaction, respectively, the initial dipole moment is quite different for the two systems and it changes from $30.6 \mathrm{fm}$ for the more charge asymmetric system, ${ }^{40} \mathrm{Ca}+{ }^{152} \mathrm{Sm}$, to $5.3 \mathrm{fm}$ for the charge symmetric one. Therefore, in this experiment all the reaction parameters were kept identical except for the initial dipole moment (as in our previous experiments). The dynamical dipole mode was investigated in both fusion-evaporation and fission events for the first time.

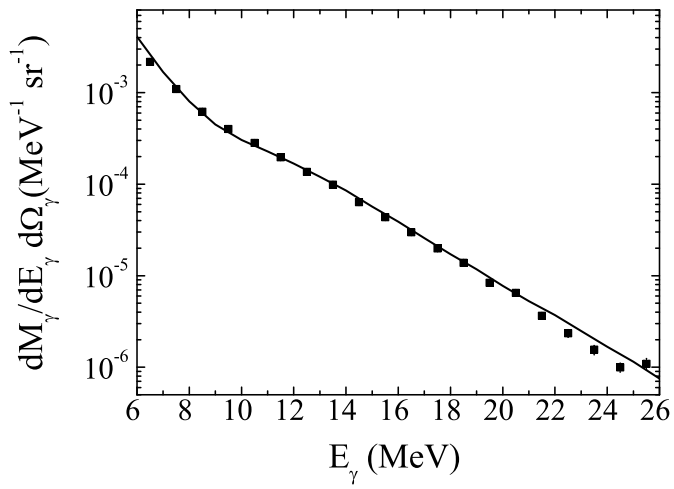

Fig. 1. $\gamma$-ray spectrum obtained in coincidence with fusion-evaporation residues for the ${ }^{48} \mathrm{Ca}+{ }^{144} \mathrm{Sm}$ reaction (symbols) compared with the theoretical spectrum calculated with the code CASCADE and folded by the response function of the experimental apparatus (line).

The $\gamma$-rays and the light charged particles were detected by using the 180 barium fluoride $\left(\mathrm{BaF}_{2}\right)$ modules of the MEDEA experimental apparatus (for details see [18]) that covers the polar angular range between $\theta_{l a b}=30^{\circ}$ and $\theta_{l a b}=170^{\circ}$ and the full range in the azimuthal angle $\phi$. The discrimination between $\gamma$-rays and light charged particles was performed by combining a pulse shape analysis of the $\mathrm{BaF}_{2}$ signal with a time of flight measurement between each $\mathrm{BaF}_{2}$ detector and the radiofrequency signal of the Cyclotron. The fusion-evaporation residues were detected by four position sensitive Parallel Plate Avalanche Counters (PPACs) located symmetrically around the beam direction at $70 \mathrm{~cm}$ from the target and cov- 
ering the angular range from $\theta=3^{\circ}$ to $10.5^{\circ}$. The fission events were identified by detecting the two kinematically coincident fission fragments with position sensitive PPACs, centered at $\theta=52.5^{\circ}$ symmetrically around the beam axis and covering the angular range from $\theta=41.9^{\circ}$ to $63.1^{\circ}$. These PPACs were positioned such that allowed the measurement of $\gamma$-rays emitted parallel $\left(0^{\circ}\right)$ or perpendicular $\left(90^{\circ}\right)$ to the spin axis of the composite system. Down-scaled single events together with coincidence events between a PPAC and at least one fired $\mathrm{BaF}_{2}$ scintillator were collected during the experiment. A coincidence event was accepted if the deposited energy in $\mathrm{BaF}_{2}$ detector was greater than $\sim 5.5 \mathrm{MeV}$ for $\gamma$-rays. The coincidence request eliminated any cosmic ray contamination of the $\gamma$-ray spectra.
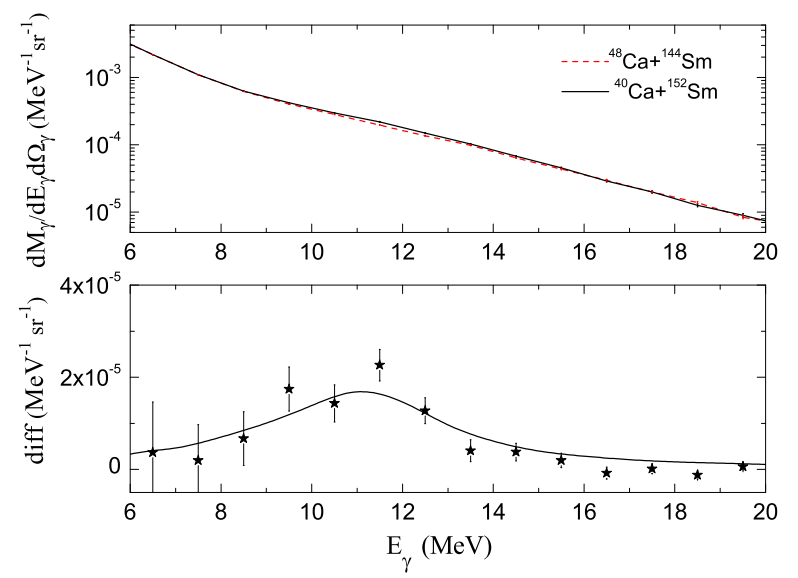

Fig. 2. Experimental $\gamma$-ray spectrum of the charge symmetric and charge asymmetric reactions (top) and their difference (bottom) for fusion-evaporation events. The solid line in the bottom is a fit of the data as described in the text.

All the collected statistics has been analyzed for $\mathrm{BaF}_{2}$ detectors in the rings situated at $\theta_{l a b}=82^{\circ}, 97^{\circ}$ and $112^{\circ}$ with respect to the beam direction while in ref. $[19,20]$ we presented preliminary results of the same experiment, concerning a smaller part of the collected statistics. In Fig. 1 the obtained experimental $\gamma$-ray spectrum for the charge symmetric reaction, ${ }^{48} \mathrm{Ca}+{ }^{144} \mathrm{Sm}$, is shown with the symbols while the theoretical spectrum calculated by means of the code CASCADE [21] and folded by the response function of the experimental apparatus [22] is displayed with the line. The parameters used in this calculation are: a $\mathrm{CN}$ mass $\mathrm{A}=192$ with an excitation energy $\mathrm{E}^{*}=236 \mathrm{MeV}$ and a level density parameter $\mathrm{a}=\mathrm{A} / 11$ $\mathrm{MeV}^{-1}$. The GDR strength function was taken to be a lorentzian curve with centroid energy $\mathrm{E}_{G D R}=13 \mathrm{MeV}$, width $\Gamma_{G D R}=12 \mathrm{MeV}$ and strength $\mathrm{S}_{G D R}=100 \%$ of the E1 energy-weighted sum-rule strength throughout the calculation. We notice that a good agreement is obtained between the data and the statical $\gamma$-ray spectrum.
In Fig. 2 we present the experimental $\gamma$-ray spectrum of the charge symmetric and charge asymmetric reactions (top) and their difference (bottom) for fusion-evaporation events while in Fig. 3 we show the same spectra for mass symmetric fission events by requiring a coincidence between the two PPACs. In both cases we notice an excess of $\gamma$-rays in the more charge asymmetric reaction that is concentrated in the energy range $8<\mathrm{E}_{\gamma}<14 \mathrm{MeV}$ (symbols in the bottom part of Fig. 2 and Fig. 3). This excess is related to the dynamical dipole mode $\gamma$ decay and was fitted by means of a lorentzian curve folded by the experimental apparatus response function (line). The considered fitting interval begins where the percent difference (not shown in the paper) between the spectra starts to raise, that is $\mathrm{E}_{\gamma}>8$ (9) $\mathrm{MeV}$ for fusion (fission) events, avoiding thus the low energy range of the data, displayed in the top of Fig. 2 and 3, with the steep exponential yield, dominated by statistical emission. The dynamical dipole mode centroid energy $\mathrm{E}_{d d}$ and width $\Gamma_{d d}$ used for the fit are: $\mathrm{E}_{d d}=11 \mathrm{MeV}$ and $\Gamma_{d d}=3.5 \mathrm{MeV}$. We are able to reproduce the difference spectrum for both evaporation and fission events with the same fit parameters, that is in agreement with what we expect for this pre-equilibrium $\gamma$-ray emission. Furthermore, we notice that, the centroid energy $\mathrm{E}_{d d}$ is lower than that of the CN GDR, $\mathrm{E}_{G D R}=13 \mathrm{MeV}$, suggesting a large deformation of the emitting source in agreement with the observation in other systems $[11,14]$. In a naive picture of two colliding nuclei at the touching configuration, we expect $\mathrm{E}_{d d} \sim \frac{78}{A_{1}^{1 / 3}+A_{2}^{1 / 3}} \sim 9 \mathrm{MeV}, A_{1}$ and $A_{2}$ being the colliding ion masses. The fact that it was found to be somewhat larger than predicted, is consistent with the expectation that some density overlap already exists at the start up of the dipole oscillation [2].

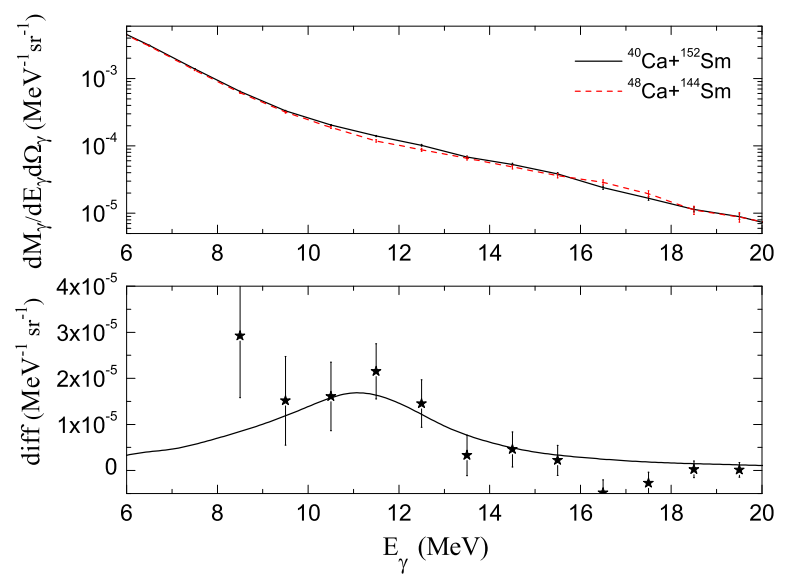

Fig. 3. Same as in Fig. 2 for mass symmetric fission events obtained by requiring a coincidence between the two PPACs.

From the above discussion we can conclude that the dynamical dipole mode survives in composite sys- 
tems in the mass region $\mathrm{A} \sim 190$ at the studied incident energy of $\sim 11 \mathrm{MeV} / \mathrm{u}$. However, its yield is comparable with that obtained for reactions leading to $\mathrm{A} \sim 130$ composite systems at incident energies ranging from 9 to $16 \mathrm{MeV} / \mathrm{u}$ but having a lower initial dipole moment. This observation supports the theoretical prediction that the heavier mass of the reaction partners causes a decrease of the dynamical dipole $\gamma$ yield. In order to draw definite conclusions, the $\gamma$-ray spectra collected at the remaining polar angles will be analyzed to extract the angular distributions for evaporation events and the $\gamma$ ray-fragment angular correlations for fission events. The experimental findings will be compared with theoretical predictions performed within a $B N V$ transport model, based on a collective bremsstrahlung analysis of the entrance channel reaction dynamics [2].

\section{Study of the dynamical dipole mode by using radioactive beams}

The different aspects of the dynamical dipole mode and its associated pre-equilibrium radiation (centroid energy, damping, angular distribution, excitation function) can be addressed in a much powerful way by combining stable and radioactive beams. The use of radioactive beams allows to attain much larger charge asymmetries than previously attained with stable ones, while the combination of radioactive and stable beams results in a very large number of target-projectile combinations that gives the possibility to perform a systematic study of the phenomenon.

The dynamical dipole radiation emitted in reactions induced by radioactive beams can be very useful to probe the density dependence of the symmetry energy in the Equation of State at nuclear densities lower than the saturation one, where the dynamical dipole mode is active [23]. The different many-body theories agree on the prediction of the symmetry energy at the saturation density but they largely diverge below and above saturation because of the highly controversial isospin dependence of the employed effective interactions. Recent theoretical calculations performed within the BNV transport model [23] for the ${ }^{132} \mathrm{Sn}+{ }^{58} \mathrm{Ni}$ reaction at $\mathrm{E}_{l a b}=10 \mathrm{MeV} / \mathrm{u}$, using either a smooth (Asysoft) or a rapid (Asystiff) decreasing function of the symmetry energy towards lower densities, show different dynamical dipole features with differences that are large enough to be discriminated experimentally. The ${ }^{132} \mathrm{Sn}+{ }^{58} \mathrm{Ni}$ reaction was chosen because of the very large entrance channel charge asymmetry that results in an initial dipole moment between the colliding ions that is twice the one attained with stable beams. From an experimental point of view and by using the radioactive beams that will be available in different facilities all over the world we could consider the reaction pair: ${ }^{132} \mathrm{Sn}+{ }^{58} \mathrm{Ni}(\mathrm{D}=45$ $\mathrm{fm})$ and ${ }^{48} \mathrm{Ca}+{ }^{142} \mathrm{Ce}(\mathrm{D}=3.13 \mathrm{fm})$ leading to the ${ }^{190} \mathrm{Pt} \mathrm{CN}$ to obtain the dynamical dipole yield employing the usual difference technique.

\section{Conclusion}

In this contribution, we presented results on the investigation of the dynamical dipole mode in reactions involving heavier mass reaction partners than those studied previously that form composite systems in the mass region of ${ }^{192} \mathrm{~Pb}$. Because of its prompt nature, the dynamical dipole mode $\gamma$ decay could represent a new cooling mechanism of the composite system, becoming thus of interest for the synthesis of superheavy elements. We show that the dynamical dipole mode survives in systems with mass $A \sim 190$, though its yield does not increase as expected with the initial dipole moment. That could be a signature of the role played by the mass of the reaction partners.

The advent of intense radioactive beams will help in maximizing the charge asymmetry between the colliding ions, shedding thus new light on different interesting aspects that could be investigated using the prompt dipole radiation as a probe, like the investigation of the symmetry energy at sub-saturation density.

\section{References}

1. Ph. Chomaz et al., Nucl. Phys. A563 (1993) 509

2. V. Baran et al., Nucl. Phys. A679 (2001) 373 and Phys. Rev. Lett. 87 (2001) 182501

3. C. Simenel et al., Phys. Rev. C76 (2007) 024609

4. Y. Aritomo, T. Wada, M. Ohta, and Y. Abe, Phys. Rev. C59 (1999) 796

5. C. Simenel et al., Phys. Rev. Lett. 86 (2001) 2971

6. D. Pierroutsakou et al., Eur. Phys. J. A16 (2003) 423

7. F. Amorini et al., Phys. Rev. C69 (2004) 014608

8. M. Papa et al., Phys. Rev. C72 (2005) 064608

9. S. Flibotte et al., Phys. Rev. Lett. 77 (1996) 1448

10. M. Cinausero et al., Il Nuovo Cimento 111 (1998) 613

11. D. Pierroutsakou et al., Eur. Phys. J. A17 (2003) 71

12. D. Pierroutsakou et al., Phys. Rev. C71 (2005) 054605

13. B. Martin et al., Phys. Lett. B664 (2008) 47

14. D. Pierroutsakou et al., Phys. Rev. C80 (2009) 024612

15. A. Corsi et al., Phys. Lett. B679 (2009) 197

16. A. Gavron, Phys. Rev. C21(1980) 230

17. M.P. Kelly et al., Phys. Rev. Lett. 82 (1999) 3404

18. E. Migneco et al., NIM A314 (1992) 31

19. C. Parascandolo et al., Nucl. Phys. A834 (2010)198c

20. D. Pierroutsakou, Int. J. Mod. Phys. E19 (2010) 1031

21. F. Puhlhofer, Nucl. Phys. A280(1977) 267, M.N. Harakeh extended version (private communication)

22. G. Bellia et al., NIM A329(1993) 173

23. V. Baran et al., Phys. Rev. C79 (2009) 021603(R) 